\title{
GRAde: a long-read sequencing approach to efficiently identifying the CYP11B1/CYP11B2 chimeric form in patients with glucocorticoid-remediable aldosteronism
}

\author{
Yu-Ching Wu' ${ }^{1}$, Chia-I Chen ${ }^{1}$, Peng-Ying Chen ${ }^{2}$, Chun-Hung Kuo ${ }^{1}$, Yi-Hsuan Hung ${ }^{1}$, Kang-Yung Peng ${ }^{2}$,

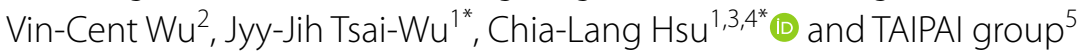

From The 19th Asia Pacific Bioinformatics Conference (APBC 2021) Tainan, Taiwan. 3-5 February 2021

\section{${ }^{*}$ Correspondence:}

jjtsai@ntuh.gov.tw

chialanghsu@ntuh.gov.tw

1 Department of Medical

Research, National Taiwan University Hospital, Taipei,

Taiwan

Full list of author information is available at the end of the article

\begin{abstract}
Background: Glucocorticoid-remediable aldosteronism (GRA) is a form of heritable hypertension caused by a chimeric fusion resulting from unequal crossing over between 11 $\beta$-hydroxylase (CYP11B1) and aldosterone synthase (CYP11B2), which are two genes with similar sequences. Different crossover patterns of the CYP11B1 and CYP11B2 chimeric genes may be associated with a variety of clinical presentations. It is therefore necessary to develop an efficient approach for identifying the differences between the hybrid genes of a patient with GRA.

Results: We developed a long-read analysis pipeline named GRAde (GRA deciphering), which utilizes the nonidentical bases in the CYP11B1 and CYP11B2 genomic sequences to identify and visualize the chimeric form. We sequenced the polymerase chain reaction (PCR) products of the CYP11B1/CYP11B2 chimeric gene from 36 patients with GRA using the Nanopore MinION device and analyzed the sequences using GRAde. Crossover events were identified for 30 out of the 36 samples. The crossover sites appeared in the region exhibiting high sequence similarity between CYP11B1 and CYP11B2, and $53.3 \%$ of the cases were identified as having a gene conversion in intron 2. More importantly, there were six cases for whom the PCR products indicated a chimeric gene, but the GRAde results revealed no crossover pattern. The crossover regions were further verified by Sanger sequencing analysis.
\end{abstract}

Conclusions: PCR-based target enrichment followed by long-read sequencing is an efficient and precise approach to dissecting complex genomic regions, such as those involved in GRA mutations, which could be directly applied to clinical diagnosis. The scripts of GRAde are available at https://github.com/hsu-binfo/GRAde.

Keywords: Glucocorticoid-remediable aldosteronism, Chimeric genes, Long-read sequencing author(s) and the source, provide a link to the Creative Commons licence, and indicate if changes were made. The images or other third party material in this article are included in the article's Creative Commons licence, unless indicated otherwise in a credit line to the material. If material is not included in the article's Creative Commons licence and your intended use is not permitted by statutory regulation or exceeds the permitted use, you will need to obtain permission directly from the copyright holder. To view a copy of this licence, visit http:// creativecommons.org/licenses/by/4.0/. The Creative Commons Public Domain Dedication waiver (http://creativecommons.org/publi cdomain/zero/1.0/) applies to the data made available in this article, unless otherwise stated in a credit line to the data. 


\section{Background}

Primary aldosteronism (PA) is the most common and curable form of secondary arterial hypertension. Most diagnosed cases of PA are mainly caused by either aldosterone overproduction by both adrenal glands or unilateral aldosterone-producing adenomas (APA) [1]. However, about $5 \%$ of cases are inherited forms of familial hyperaldosteronism $(\mathrm{FH})$ [2]. According to current understanding, there are three well-established forms of FH (FH-I-III), and some germline mutations associated with PA, such as CACN1H and CACN1D, have been identified [3]. However, data from genetic analyses reveal a more complex situation, and more heritable forms of PA may still be undiscovered [2].

Familial hyperaldosteronism type I (FH1), also called glucocorticoid-remediable aldosteronism (GRA), is transmitted as an autosomal-dominant disorder and accounts for $0.5-1.0 \%$ of PA cases [4]. GRA is caused by a chimeric gene resulting from a nonhomologous crossing-over event on chromosome 8q24.3, between the 11ß-hydroxylase (CYP11B1) and aldosterone synthase (CYP11B2) genes. This chimeric enzyme contains the promoter of CYP11B1 at the $5^{\prime}$ end and the coding sequences from CYP11B2 at the $3^{\prime}$ end. It can therefore synthesize aldosterone under adrenocorticotropic hormone (ACTH) control (Fig. 1A).

Although GRA is a genetic disease, the clinical and biochemical characteristics of patients are highly variable, and even patients from the same familial type may present different symptoms [5, 6]. In addition, different crossover patterns of the chimeric CYP11B1/CYP11B2 gene within a familial type have been described [7]. These findings

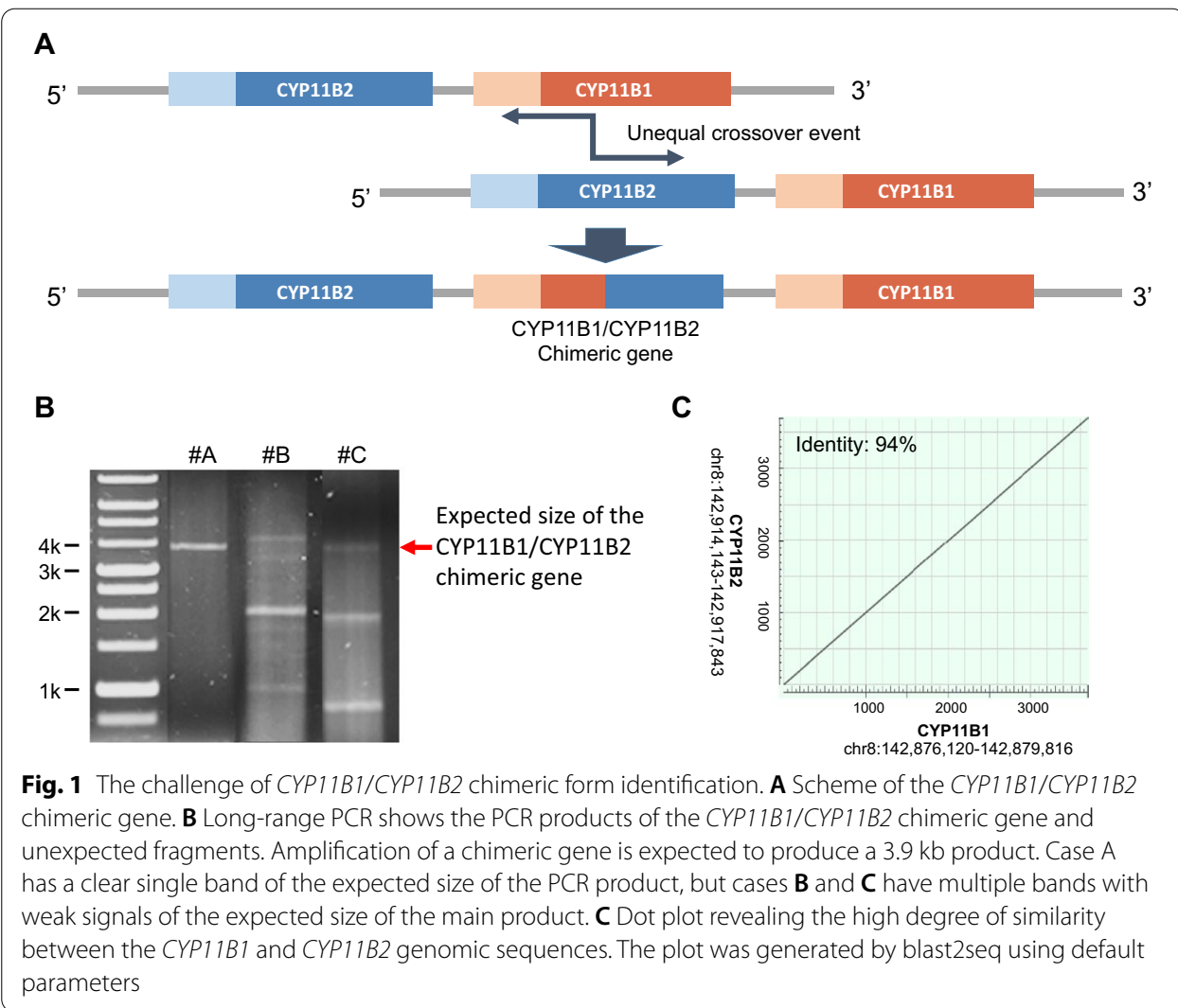


suggest that variability in clinical presentations might be related to heterogeneity in the hereditary factor-in particular, in the crossover pattern of the hybrid gene. Currently, the genetic diagnosis of GRA is made using Southern blotting or long-range polymerase chain reaction (PCR) techniques [8-12]. However, these methods are unable to precisely determine the crossover pattern. Therefore, it is crucial to develop a more precise method for identifying the specific type of hybrid gene that is carried by a patient.

The detection of pathogenic gene fusion in inherited diseases and oncology is particularly useful. In general, fusion events cause a loss or gain of function in one of the fused partners. However, unlike with oncogenic fusion genes, it is relatively difficult to detect gene conversion in genes with highly similar sequences. New detection strategies for this kind of gene fusion are urgently required to facilitate diagnostic and therapeutic decisions.

Since the introduction of next-generation sequencing (NGS), tremendous progress has been achieved in all fields of biology. The declining costs and growing availability of NGS have made it the method of choice for genetic analysis and related applications. Nevertheless, NGS suffers from a limited availability of data on several aspects, such as repetitive elements, polymorphic regions, camouflaged genes, and large structural variations (SVs), which prevents full extraction of information associated with the genome $[13,14]$. However, recently developed single-molecule sequencing techniques such as single-molecule real-time sequencing (SMRT) and nanopore sequencing provide access to larger variations, because the read lengths are typically several thousands of bases $[15,16]$. These techniques provide the opportunity to investigate or diagnose diseases caused by pathogenic structural variations and gene conversion, such as GRA. In this study, we used Oxford nanopore technology (ONT) to sequence the PCR products of chimeric $C Y P 11 B 1 / C Y P 11 B 2$ genes and developed a long-read analysis pipeline that can efficiently identify and visualize the chimeric forms.

\section{Results}

\section{The challenge of GRA chimeric form identification}

Previous studies have suggested that a variety of CYP11B1/CYP11B2 chimeric forms are associated with different clinical presentations [5, 6]. Long-range PCR is one of the standard approaches to GRA diagnosis. However, in a few of GRA cases, multiple bands with weak signals for the expected PCR products are obtained (Fig. 1B), making diagnosis difficult. To avoid misdiagnosis of patients with GRA, it is crucial to identify the exact crossover site. The current method for identifying crossover sites is multiplex PCR with Sanger sequencing [12]. However, the genomic sequences of CYP11B1 and CYP11B2 are about $94 \%$ identical in the main crossover region, making primer design for the enrichment of regions that are specific to only one of the two genes challenging (Fig. 1C). In addition, this procedure is time-consuming and laborious. To address this issue, we proposed an alternative strategy that combines long-range PCR with nanopore sequencing to confirm the chimeric gene and identify the crossover site, and we developed an analysis pipeline to decipher these long-read sequences. 


\section{Overview of GRAde}

The analysis workflow of GRAde is illustrated in Fig. 2A. The input for GRAde was FASTQ files, which were generated from the long sequencing reads of PCR products derived from GRA samples. This pipeline consists of two components: quality control for the sequencing reads, and the procedure for determining the crossover regions of the $C Y P 11 B 1 / C Y P 11 B 2$ chimeric form. High-quality reads were obtained by correcting them using a nonhybrid approach, Canu [17], and then mapping them to the human reference genome. Only the reads that aligned with the loci of $C Y P 11 B 1$ and $C Y P 11 B 2$ were considered for further analysis. To accurately dissect each qualifying read, GRAde used the Smith-Waterman algorithm to align reads to the CYP11B1 and CYP11B2 genomic sequences, respectively, and then analyzed the alignment results to identify the crossover region.

Due to the high degree of sequence similarity between the CYP11B1 and CYP11B2 genes, we first identified the "discriminating" and "ambiguous" bases by comparing them to the CYP11B1 (chr8:142876120-142879816) and CYP11B2 (chr8:142914143142917843) sequences (Fig. 2B). We then used the discriminating bases to distinguish the sequences from the genomic source (Fig. 2C). There were 180 and 188 discriminating bases for $C Y P 11 B 1$ and $C Y P 11 B 2$, respectively (Additional file 1). The median of the positional distribution of the neighbor discriminating bases was $11 \mathrm{bp}$, with a range of 1-221 bp. Ideally, the ambiguous bases should perfectly match both genes, but there are typically some mismatches due to errors in the PCR or sequencing processes and genetic polymorphisms (Fig. 2C). Mismatches of ambiguous bases may affect the interpretation,

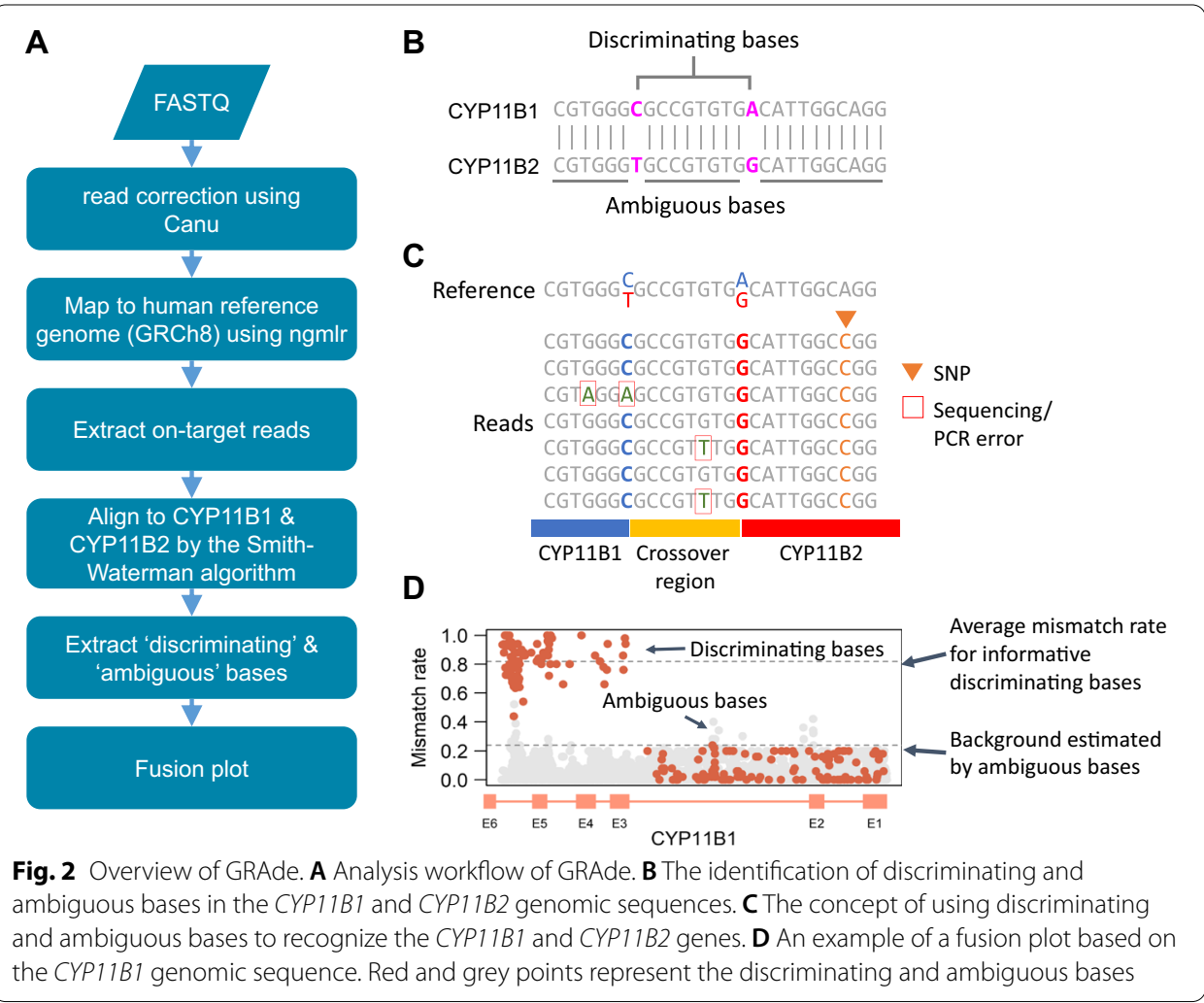


so the ambiguous bases were discarded if polymorphisms were reported at these positions in the single-nucleotide polymorphism (SNP) database (dbSNP). Based on the alignment results of all reads mapped to $C Y P 11 B 1$ and $C Y P 11 B 2$, we calculated the mismatch rates of each discriminating and ambiguous base and visualized them as a fusion plot (Fig. 2D). Because of the relatively high error rate of nanopore sequencing, if the bases aligned to discriminating sites did not exactly match either CYP11B1 or CYP11B2, we considered them to be sequencing or PCR errors and did not include them in the fusion plot. This fusion plot provides the intuitive and interpretable chimeric form for each sample.

\section{Variety of CYP11B1/CYP11B2 chimeric forms in GRA samples}

We collected 36 samples from patients who were diagnosed with GRA based on clinical practice guidelines, which was confirmed by using the long-range PCR technique to reveal the chimeric genes. The chimeric genes were amplified using the long-range PCR technique and then subjected to nanopore sequencing. We also amplified CYP11B2 genes from six other patients with PA as negative controls. The results of the GRAde analysis of the 36 GRA samples are summarized in Table 1, and the fusion forms are shown in Fig. 3A and Additional file 2. Sixteen of the 36 cases had fusion sites located at intron 2 , and the crossover region of the other cases was within the ranges of exon 3-intron 3 (seven cases), exon 4-intron 4 (five cases) and exon 5-intron 5 (two cases). There were no fusion patterns in the fusion plots for

Table 1 GRAde analysis results for GRA samples

\begin{tabular}{|c|c|c|c|}
\hline Sample no. & Crossover region & Sample no. & Crossover region \\
\hline \#1 & $E 2-12$ & $\# 19$ & $E 2-12$ \\
\hline \#2 & E3-13 & $\# 20$ & $E 2-12$ \\
\hline \#3 & $E 2-12$ & \#21 & E4-14 \\
\hline \#4 & E3-13 & $\# 22$ & No fusion observed \\
\hline$\# 5$ & $E 2-12$ & $\# 23$ & No fusion observed \\
\hline \#6 & E3-13 & $\# 24$ & No fusion observed \\
\hline \#7 & $E 2-12$ & \#25 & E2-12 \\
\hline \#8 & E3-13 & $\# 26$ & E3-13 \\
\hline \#9 & E3-I3 & \#27 & E4-14 \\
\hline \#10 & E3-13 & \#28 & $E 2-12$ \\
\hline$\# 11$ & $E 2-12$ & $\# 29$ & No fusion observed \\
\hline$\# 12$ & E5-15 & $\# 30$ & $E 2-12$ \\
\hline$\# 13$ & No fusion observed & $\# 31$ & E5-15 \\
\hline$\# 14$ & No fusion observed & $\# 32$ & $E 4-14$ \\
\hline \#15 & $E 2-12$ & \#33 & $E 2-12$ \\
\hline \#16 & $E 2-12$ & \#34 & $E 2-12$ \\
\hline$\# 17$ & $E 2-12$ & \#35 & $E 2-12$ \\
\hline \#18 & $\mathrm{E} 4-14$ & \#36 & $\mathrm{E} 4-14$ \\
\hline
\end{tabular}

E2-12: located in exon 2 and intron 2 E3-13: located in exon 3 and intron 3 E4-14: located in exon 4 and intron 4 E5-15: located in exon 5 and intron 5 No fusion observed: no clear fusion pattern was observed in the GRAde fusion plot 


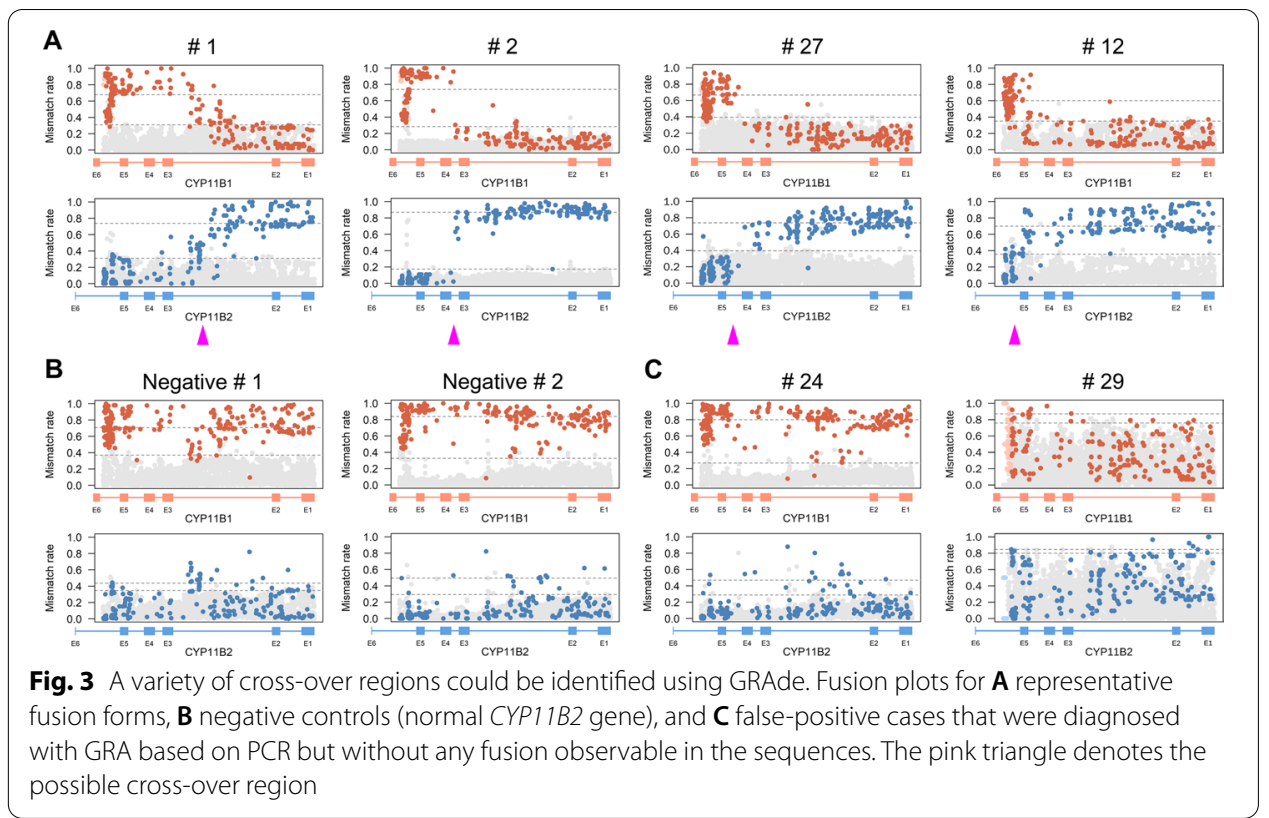

any of the negative control samples of normal CYP11B2 genes (Fig. 3B). Notably, there were also six GRA cases for whom no fusion patterns were apparent (Fig. 3C and Additional file 2), suggesting that their diagnoses might be based on false-positive test results.

\section{Validating the crossover site via multiplex PCR with Sanger sequencing}

To demonstrate the validity of our approach and GRAde, we selected a case for whom the PCR produced multiple products and the signal for the chimeric gene was relatively weak (Fig. 4A). After sequencing the PCR products using the nanopore technology and analyzing the reads using GRAde, a clear fusion pattern was revealed in the fusion plot, although the background noise was high at the $3^{\prime}$ end of the chimeric gene (Fig. 4B). Using the fusion plot, we designed primers to amplify the identified crossover region and sequenced the amplified products via Sanger sequencing. Nucleotide sequence analysis showed that the gene-conversion site was in the middle of intron 2 , which was consistent with the GRAde result.

\section{Runtime and robustness of GRAde}

We evaluated the runtime and robustness of GRAde for use as a diagnostic tool. We generated testing sets that were randomly sampled from case No. 34, with a varying number of reads $(100,200,500,1000,2000$, or 3000). As shown in Additional file 3, analysis of input with 3000 reads could be completed within six minutes, and the most time-consuming step was performing the Canu algorithm for hybridizationbased sequence correction. We were able to achieve stable results using the sample with 200 reads, so we consider that to be the minimum number of reads required for analysis (Additional file 3). 
A

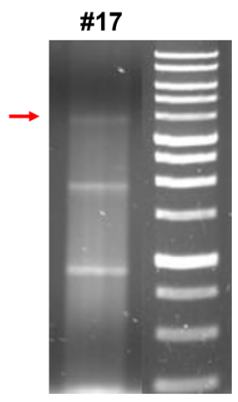

C

CYP11B1 CAAGTCCAGAAGGCTGGGTGCACATAACGGAAGGAAGGGGAGCGCACCTGTATGTGTGTGCGTCTTGCAT Chimeric gene CAAGTCCAGAAGGCTGGGTGCACATAACGGAAGGAAGGGGAGCGCACCTGTATGTGTGTGTGTCTTGCAT CYP11B2 CAGGTCCAGGAGGCTGGGTGCGCATAATGGAAGGAAGGGGAGCGCTCCTGTATGTGTGTGTGTCTTGCAT

CAAGTCCA GAAGCTGGGTGCACATAACGGAAGAAGGGGAGCGCACCTGTATGTGTGIGTGTCTTGCAT

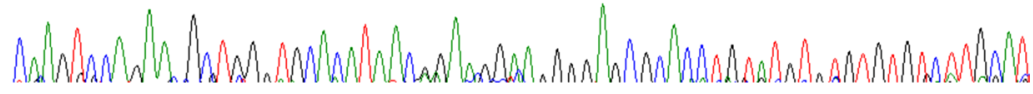

Fig. 4 A case in which the cross-over region was validated by Sanger sequence analysis. A Long-range PCR revealed PCR products of the CYP11B1/CYP11B2 chimeric gene in sample \#17. Amplification with a chimeric gene is expected to produce a $3.9 \mathrm{~kb}$ product. B The fusion plot of sample \#17 reveals a possible cross-over region located in intron 2, with high background noise. $\mathbf{C}$ Sequencing of the chimeric PCR product demonstrated that the crossover site was located in intron 2. The nucleotides that differed between CYP11B1, CYP11B2, and the chimeric gene are highlighted in different colors

\section{Discussion}

The emerging long-read sequencing technologies offer improvements in the characterization of genetic variation and regions that are difficult to assess using short reads. These techniques have been used to investigate genetic disorders with previously known or strongly suspected disease loci [18] and are considered a diagnostic tool. The general strategy of long-read-based diagnosis is to enrich the locus of causality and then perform long-read sequencing. Long-range PCR is a technique currently used for the detection of GRA [8-12], so we chose it as the enrichment method. However, GRA is a special case because the locus of causality is formed by two genes with a high degree of sequence similarity (Fig. 1C). This results in challenging primer design and poor PCR products for evaluation, due to issues like the presence of multiple bands and weak signals for the expected chimeric products (Fig. 1B). Also, the presence of the expected band in the PCR product does not necessarily mean it is a chimeric gene, which can lead to incorrect diagnoses based on false-positive test results (Fig. 3C). Therefore, long-range PCR in addition to long-read sequencing is a more efficient method of chimeric gene detection than traditional Sanger sequencing and may reduce the false-positive rate of traditional PCR testing.

However, PCR-based enrichment still has several limitations. PCR amplification can be influenced by improper PCR conditions $[19,20]$, thus producing false negatives or incorporating PCR errors. Analysis of sequencing reads from PCR products with multiple bands may also be hampered by a high level of background noise and an unclear fusion pattern (Fig. 3A). To avoid this, the development of alternative 
target-enrichment methods for GRA chimeric genes is required, such as capturebased [21, 22] and CRISPR-based enrichment methods [23, 24].

In our GRA cohort, there were six patients who had PCR products for sequencing in which we could identify no fusion pattern. The possible reasons for this include poor integrity and low purity of the genomic DNA, which could have resulted from the state of storage and the process of DNA extraction. Other possible factors include poor specificity of the primers, inappropriate DNA input, and nucleic-acid contamination. It is difficult to design extremely specific primers for the crossover region of chimeric genes, so this pipeline can assist in excluding nonspecific PCR products and distinguishing the correct signal.

In addition to the CYP11B1/CYP11B2 chimeric gene, there are some SNPs at the $C Y P 11 B 1$ and $C Y P 11 B 2$ loci that are also associated with hypertension [25]. Theoretically, these single nucleotide variants (SNV) can be detected in the current longread sequence data. To provide precise diagnosis and treatment of GRA, however, it is critical to report all possible variants in genetic testing. Although GRAde was developed for the identification of chimeric forms, therefore, we will incorporate the function of variant-calling to identify SNVs and small insertions and deletions (indels).

Although GRAde is specifically designed for the identification of crossover sites between CYP11B1 and CYP11B2 in GRA patients, the analysis strategy used in GRAde could also be applied to other diseases that are caused by dysfunctional proteins due to unequal crossover events. For example, chronic granulomatous disease (CGD) is caused by the chimeric form of NCF1 (neutrophil cytosolic factor 1) and its pseudogenes $N C F 1 B$ (neutrophil cytosolic factor 1B pseudogene) and NCF1C (neutrophil cytosolic factor 1C pseudogene) [26]. Because these two pseudogenes are on either side of $N C F 1$ and have $99 \%$ sequence identity to $N C F 1$, distinguishing NCF1 from its pseudogenes in CGD patients relies on a set of SNPs [27] and an analysis similar in concept to that of GRAde. In addition to CGD, several other diseases are caused by the chimeric products of the crossover between a gene and its pseudogenes, such as congenital adrenal hyperplasia, caused by the chimeric genes CYP21A1P/CYP21A2 [28], and Gaucher disease, caused by a fusion gene formed from $G B A$ (glucosylceramidase beta) and its pseudogene GBAP1 (glucosylceramidase beta pseudogene 1) [29]. GRAde could easily be modified and used for crossover-site identification in other diseases. Besides the detection of specific fusion genes, our strategy could be also applied to genome-wide detection of this kind of gene fusion by systematically identifying discriminating bases in homologous genes.

\section{Conclusions}

In this study, we proposed the strategy of combining long-range PCR with long-read sequencing techniques to identify gene conversions, such as the one that causes GRA. This approach is not only more efficient than general multiplex PCR followed by Sanger sequencing, but also reduces the false-positive rate for PCR-based genetic testing. This analysis procedure could be applied to the diagnosis of other diseases caused by unequal crossover between two genes with highly similar sequences. 


\section{Methods}

Patients

This study was approved by the institutional review board of the National Taiwan University Hospital, Taipei, Taiwan (No. 200611031R) (ClinicalTrials.gov number NCT00746070). All participants provided written informed consent before inclusion in the study. The Taiwan Primary Aldosteronism Investigation (TAIPAI) group enrolled possible PA patients who first had their aldosterone-to-renin ratio (ARR) screened for PA detection and were then followed-up. Screening, confirmation, and subtype identification of the PA were performed in hypertensive patients according to the standard TAIPAI protocol and aldosteronism consensus [30-33]. Fulfillment of the following three conditions confirmed a diagnosis of PA: (1) autonomous excess aldosterone production evidenced by an ARR > 35; (2) a TAIPAI score [34] of >60\%; (3) seated post-saline loading plasma aldosterone concentration (PAC) $>16 \mathrm{ng} / \mathrm{dL}$ [35], or PAC/plasma renin activity $(\mathrm{PRA})>35(\mathrm{ng} / \mathrm{dL}) /(\mathrm{ng} / \mathrm{mL} / \mathrm{h})$ in a post-captopril/ losartan test [30].

\section{Sample preparation}

For the detection of the chimeric gene, PCR was performed using the method described by MacConnachie et al. [12] with some modifications. We used the following primer sets to amplify the normal $C Y P 11 B 2$ gene and the chimeric $C Y P 11 B 1 / C Y P 11 B 2$ gene with PfuUltra II Fusion HS DNA Polymerase (Agilent): forward: 5'CAGGTCCAGAGCCAG TTCTCCCAT/reverse: 5'ACCCTCCTTCTCCTTGTACACCCA forward: 5'CAGTTC TCCCATGACGTGATCCCT/reverse: 5'ACCCTCCTTCTCCTTGTACACCCA.

The touchdown PCR process was as follows: $95^{\circ} \mathrm{C}$ for $2 \mathrm{~min}$; 38 cycles of denaturation at $95{ }^{\circ} \mathrm{C}$ for $1 \mathrm{~min}$; annealing at $70-61{ }^{\circ} \mathrm{C}$ for $1 \mathrm{~min}$; and extension at $72{ }^{\circ} \mathrm{C}$ for $5 \mathrm{~min}$ and $72{ }^{\circ} \mathrm{C}$ for $3 \mathrm{~min}$. The annealing temperature began at $70{ }^{\circ} \mathrm{C}$ and was lowered by $1{ }^{\circ} \mathrm{C}$ every two cycles until it reached $61^{\circ} \mathrm{C}$; this annealing temperature was maintained until the end of the cycling process. The PCR amplicons were evaluated in a 0.8\% agarose gel, cleaned up with the 0.45X Agencourt AMPure XP beads (Beckman Coulter), and quantified using a Qubit fluorometer (Life Technologies).

\section{Sample barcoding}

For simultaneous detection of multiple samples, we used the ligation method to tag the amplified sample with the native barcoding adaptor (Oxford Nanopore Technologies), which allows up to 24 different libraries (barcodes $1-24$ ) to be combined and loaded onto a single flow cell at the same time. The amplified fragment end was repaired and dA-tailed using the End Repair/dA-tailing Module (KAPA Roche). The end-repaired product was purified using 1X Agencourt AMPure XP beads. Next, a unique dT-tailed barcode adaptor was ligated on the dA-tailed template using ligation Master Mix (KAPA Roche). The barcoded samples were then purified with $0.45 \mathrm{X}$ Agencourt AMPure XP beads. The quality and quantity of each sample were evaluated using Nanodrop and a Qubit fluorometer, respectively. The barcoded samples were equally pooled for the sequencing library preparation. 


\section{Library preparation and sequencing}

For the construction of the sequencing library, we used the KAPA hyper prep kit (Roche). First, the amplified and barcoded samples were pooled together, and end repair and dA-tailing were performed using the End Repair/dA-tailing Module. The end-repaired product was purified using 1X Agencourt AMPure XP beads. Next, adapter ligation and tethering were carried out with sequencing adapter (Oxford Nanopore Technologies) and ligation Master Mix. The sequencing-adapter-ligated DNA library was then purified with 0.5X Agencourt AMPure XP beads, Adapter Bead binding buffer (Oxford Nanopore Technologies) was added, and the samples were eluted in the Elution Buffer (Oxford Nanopore Technologies).

Before sequencing, the sequencing-adapter-ligated DNA library was mixed with Library Loading beads, and SpotON Flow Cells (R9.4) (FLO-MIN106D) were primed with running buffer. The samples were run on a MinION sequencing device for approximately $24 \mathrm{~h}$, and the sequencing runs were operated by the MinKNOW software. Base-calling from the electrical data generated by the sequencer was performed using Guppy (v3.0.3) and the resulting raw sequence data were demultiplexed using qcat (v1.1.0).

\section{Implementation of GRAde}

Reads with lengths of 3000-5000 bp were considered for downstream analysis. Reads were corrected using a nonhybrid-based approach Canu (v1.4) [17] with the default parameters altered only as follows: genomeSize $=5 \mathrm{k}$, overlapper $=$ mhap, utgReAlign $=$ true, and stopOnReadQuality $=$ false. Corrected reads were compared to the human reference genome (GRCh38) using ngmlr (v0.2.7) using default parameters [36]. The reads that aligned on the loci of $C Y P 11 B 1$ and $C Y P 11 B 2$ were defined as on-target reads. Each read was aligned to the conserved regions of $C Y P 11 B 1$ and $C Y P 11 B 2$ using the Smith-Waterman algorithm, implemented by the SSW library [37]. The discriminating and ambiguous bases were extracted from the alignment between CYP11B1 and CYP11B2 by the Smith-Waterman algorithm. The mismatch rate of each discriminating and ambiguous base was calculated by parsing the alignment results of reads aligned to CYP11B1 and CYP11B2. The background noise was defined as the 99th percentile of the mismatch rate of all ambiguous bases, which is represented by the lower dashed line in the fusion plot. The foreground, represented by the upper dashed line in the fusion plot, was the average of the mismatch rates of the discriminating bases that had mismatch rates higher than the background. In addition, the mismatch rates of the discriminating bases were fitted using a sigmoid function to identify the crossover site, using the drm function implemented in the drc R package. All scripts were implemented in Python (v2.7) and R (v3.5.1).

\section{Abbreviations}

ACTH: Adrenocorticotropic hormone; APA: Aldosterone-producing adenomas; ARR: Aldosterone-to-renin ratio; CGD: Chronic granulomatous disease; CRISPR: Clustered regularly interspaced short palindromic repeat; FH: Familial hyperaldosteronism; GRA: Glucocorticoid-remediable aldosteronism; Indel: Insertion and deletion; NGS: Next-generation sequencing; ONT: Oxford Nanopore Technology; PA: Primary aldosteronism; PAC: Plasma aldosterone concentration; PCR: Polymerase chain reaction; PRA: Plasma renin activity; SMRT: Single-molecule real-time sequencing; SNP: Single-nucleotide polymorphism; SNV: Single nucleotide variant; SV: Structural variation; TAIPAI: The Taiwan Primary Aldosteronism Investigation. 


\section{Supplementary Information}

The online version contains supplementary material available at https://doi.org/10.1186/s12859-022-04561-w.

Additional file 1: Nucleotides that differed between CYP11B1 and CYP11B2.

Additional file 2: Fusion plots for all GRA patients.

Additional file 3: Runtime and robustness of GRAde.

\section{Acknowledgements}

The authors thank the National Center for High-performance Computing for computer time and facilities. We also thank the Second Core Lab and Sequencing Core, Department of Medical Research, National Taiwan University Hospital for providing laboratory facilities. We would like to thank Uni-edit (www.uni-edit.net) for editing and proofreading this manuscript.

\section{About this supplement}

This article has been published as part of BMC Bioinformatics Volume 22 Supplement 10 2021: Selected articles from the 19th Asia Pacific Bioinformatics Conference (APBC 2021): bioinformatics. The full contents of the supplement are available at https://bmcbioinformatics.biomedcentral.com/articles/supplements/volume-22-supplement-10.

\section{Authors' contributions}

YCW, JJTW, and CLH designed the study. YCW, CIC, PYC, CHK, and KYP performed the experiments. YHH and CLH implemented the tool and performed the analysis. VCW provided sample materials and clinical information. YCW, KYP, VCW, JJTW, and CLH interpreted the data. YCW and CLH drafted the manuscript. All authors read, revised, and approved the manuscript.

\section{Funding}

This work was supported by the Ministry of Science and Technology, Taiwan (MOST 107-2314-B-002-254-MY3, MOST1102221-E-002-129-MY3 to CLH) and National Taiwan University Hospital, Taipei, Taiwan (108-N4206 to YCW; 109-N4491 to $\mathrm{CIC} ; 110-\mathrm{S} 4909$ and 110-O01 to CLH). The funders did not play any role in the design of the study, the collection, analysis, and interpretation of data, or in writing of the manuscript. Publication costs are funded by National Taiwan University Hospital (110-S4909 and 110-001).

Availability of data and materials

The scripts used for the analyses have been deposited at GitHub and are available at https://github.com/hsu-binfo/ GRAde.

\section{Declarations}

\section{Ethics approval and consent to participate}

This study was conducted in accordance with the mandates of the Helsinki Declaration and the guidelines of the ethics committee of National Taiwan University Hospital. All participants provided written informed consent before inclusion in the study.

\section{Consent for publication}

Not applicable.

\section{Competing interests}

The authors declare that they have no competing interests.

\section{Author details}

${ }^{1}$ Department of Medical Research, National Taiwan University Hospital, Taipei, Taiwan. ${ }^{2}$ Department of Internal Medicine, National Taiwan University Hospital, Taipei, Taiwan. ${ }^{3}$ Graduate Institute of Oncology, National Taiwan University College of Medicine, Taipei, Taiwan. ${ }^{4}$ Graduate Institute of Medical Genomics and Proteomics, National Taiwan University College of Medicine, Taipei, Taiwan. ${ }^{5}$ TAIPAI, Taiwan Primary Aldosteronism Investigator Group and TSA, Taiwan Society of Aldosteronism, Taipei, Taiwan.

Received: 9 December 2021 Accepted: 3 January 2022

Published online: 10 January 2022

\section{References}

1. Farrugia FA, Zavras N, Martikos G, Tzanetis P, Charalampopoulos A, Misiakos EP, Sotiropoulos D, Koliakos N. A short review of primary aldosteronism in a question and answer fashion. Endocr Regul. 2018:52(1):27-40.

2. Perez-Rivas $L G$, Williams $T A$, Reincke $M$. Inherited forms of primary hyperaldosteronism: new genes, new phenotypes and proposition of a new classification. Exp Clin Endocrinol Diabetes. 2019;127(2-03):93-9.

3. Zennaro MC, Boulkroun S, Fernandes-Rosa F. An update on novel mechanisms of primary aldosteronism. J Endocrinol. 2015:224(2):R63-77 
4. Mulatero P, Tizzani D, Viola A, Bertello C, Monticone S, Mengozzi G, Schiavone D, Williams TA, Einaudi S, La Grotta A, et al. Prevalence and characteristics of familial hyperaldosteronism: the PATOGEN study (Primary Aldosteronism in TOrino-GENetic forms). Hypertension. 2011;58(5):797-803.

5. Aglony M, Martinez-Aguayo A, Carvajal CA, Campino C, Garcia H, Bancalari R, Bolte L, Avalos C, Loureiro C, Trejo P, et al. Frequency of familial hyperaldosteronism type 1 in a hypertensive pediatric population: clinical and biochemical presentation. Hypertension. 2011;57(6):1117-21.

6. Lin YF, Peng KY, Chang CH, Hu YH, Wu VC, Chueh JS, Wu KD. Adrenalectomy completely cured hypertension in patients with familial hyperaldosteronism type I who had somatic KCNJ5 mutation. J Clin Endocrinol Metab. 2019;104(11):5462-6.

7. Carvajal CA, Campino C, Martinez-Aguayo A, Tichauer JE, Bancalari R, Valdivia C, Trejo P, Aglony M, Baudrand R, Lagos $\mathrm{CF}$, et al. A new presentation of the chimeric CYP11B1/CYP11B2 gene with low prevalence of primary aldosteronism and atypical gene segregation pattern. Hypertension. 2012;59(1):85-91.

8. Malagon-Rogers M. Non-glucocorticoid-remediable aldosteronism in an infant with low-renin hypertension. Pediatr Nephrol. 2004;19(2):235-6.

9. Vonend O, Altenhenne C, Büchner NJ, Dekomien G, Maser-Gluth C, Weiner SM, Sellin L, Hofebauer S, Epplen JT, Rump LC. A German family with glucocorticoid-remediable aldosteronism. Nephrol Dial Transplant Off Publ Eur Dial Transpl Assoc Eur Ren Assoc. 2007;22(4):1123-30.

10. Fallo F, Pilon C, Williams TA, Sonino N, Morra Di Cella S, Veglio F, De lasio R, Montanari P, Mulatero P. Coexistence of different phenotypes in a family with glucocorticoid-remediable aldosteronism. J Hum Hypertens. 2004;18(1):47-51.

11. Fardella CE, Pinto M, Mosso L, Gómez-Sánchez C, Jalil J, Montero J. Genetic study of patients with dexamethasone-suppressible aldosteronism without the chimeric CYP11B1/CYP11B2 gene. J Clin Endocrinol Metab. 2001;86(10):4805-7.

12. MacConnachie AA, Kelly KF, McNamara A, Loughlin S, Gates LJ, Inglis GC, Jamieson A, Connell JM, Haites NE. Rapid diagnosis and identification of cross-over sites in patients with glucocorticoid remediable aldosteronism. J Clin Endocrinol Metab. 1998;83(12):4328-31.

13. Treangen TJ, Salzberg SL. Repetitive DNA and next-generation sequencing: computational challenges and solutions. Nat Rev Genet. 2011;13(1):36-46.

14. Ebbert MTW, Jensen TD, Jansen-West K, Sens JP, Reddy JS, Ridge PG, Kauwe JSK, Belzil V, Pregent L, Carrasquillo MM, et al. Systematic analysis of dark and camouflaged genes reveals disease-relevant genes hiding in plain sight. Genome Biol. 2019;20(1):97.

15. Huddleston J, Chaisson MJP, Steinberg KM, Warren W, Hoekzema K, Gordon D, Graves-Lindsay TA, Munson KM, Kronenberg ZN, Vives L, et al. Discovery and genotyping of structural variation from long-read haploid genome sequence data. Genome Res. 2017:27(5):677-85.

16. Norris AL, Workman RE, Fan Y, Eshleman JR, Timp W. Nanopore sequencing detects structural variants in cancer. Cancer Biol Ther. 2016;17(3):246-53.

17. Koren S, Walenz BP, Berlin K, Miller JR, Bergman NH, Phillippy AM. Canu: scalable and accurate long-read assembly via adaptive k-mer weighting and repeat separation. Genome Res. 2017;27(5):722-36.

18. Mantere T, Kersten S, Hoischen A. Long-read sequencing emerging in medical genetics. Front Genet. 2019;10:426.

19. Grunenwald H. Optimization of polymerase chain reactions. In: Bartlett JMS, Stirling D, editors. PCR protocols. Totowa: Humana Press; 2003. p. 89-99.

20. Lorenz TC. Polymerase chain reaction: basic protocol plus troubleshooting and optimization strategies. J Vis Exp. 2012;63:e3998.

21. Peng Z, Paudel D, Wang L, Luo Z, You Q, Wang J. Methods for target enrichment sequencing via probe capture in legumes. Methods Mol Biol. 2020;2107:199-231.

22. Eckert S, Chan J, Houniet D, Breuer J, Speight G. Enrichment by hybridisation of long DNA fragments for Nanopore sequencing. Microbial Genomics. 2016;2:e000087.

23. Kang S-H, Lee W, An J-H, Lee J-H, Kim Y-H, Kim H, Oh Y, Park Y-H, Jin YB, Jun B-H, et al. Prediction-based highly sensitive CRISPR off-target validation using target-specific DNA enrichment. Nat Commun. 2020;11(1):3596.

24. Gilpatrick T, Lee I, Graham JE, Raimondeau E, Bowen R, Heron A, Downs B, Sukumar S, Sedlazeck FJ, Timp W. Targeted nanopore sequencing with Cas9-guided adapter ligation. Nat Biotechnol. 2020;38(4):433-8.

25. Alvarez-Madrazo S, Mackenzie SM, Davies E, Fraser R, Lee WK, Brown M, Caulfield MJ, Dominiczak AF, Farrall M, Lathrop M, et al. Common polymorphisms in the CYP11B1 and CYP11B2 genes: evidence for a digenic influence on hypertension. Hypertension. 2013;61(1):232-9.

26. Hayrapetyan A, Dencher PC, van Leeuwen K, de Boer M, Roos D. Different unequal cross-over events between NCF1 and its pseudogenes in autosomal p47(phox)-deficient chronic granulomatous disease. Biochim Biophys Acta. 2013;1832(10):1662-72.

27. Chanock SJ, Roesler J, Zhan S, Hopkins P, Lee P, Barrett DT, Christensen BL, Curnutte JT, Gorlach A. Genomic structure of the human p47-phox (NCF1) gene. Blood Cells Mol Dis. 2000;26(1):37-46.

28. Coeli FB, Soardi FC, Bernardi RD, de Araujo M, Paulino LC, Lau IF, Petroli RJ, de Lemos-Marini SH, Baptista MT, GuerraJunior G, et al. Novel deletion alleles carrying CYP21A1P/A2 chimeric genes in Brazilian patients with 21-hydroxylase deficiency. BMC Med Genet. 2010;11:104.

29. Cormand B, Diaz A, Grinberg D, Chabas A, Vilageliu L. A new gene-pseudogene fusion allele due to a recombination in intron 2 of the glucocerebrosidase gene causes Gaucher disease. Blood Cells Mol Dis. 2000;26(5):409-16.

30. Wu VC, Hu YH, Er LK, Yen RF, Chang CH, Chang YL, Lu CC, Chang CC, Lin JH, Lin YH, et al. Case detection and diagnosis of primary aldosteronism: the consensus of Taiwan Society of Aldosteronism. J Formos Med Assoc. 2017;116(12):993-1005.

31. Wu VC, Chang HW, Liu KL, Lin YH, Chueh SC, Lin WC, Ho YL, Huang JW, Chiang CK, Yang SY, et al. Primary aldosteronism: diagnostic accuracy of the losartan and captopril tests. Am J Hypertens. 2009;22(8):821-7.

32. Kuo CC, Wu VC, Huang KH, Wang SM, Chang CC, Lu CC, Yang WS, Tsai CW, Lai CF, Lee TY, et al. Verification and evaluation of aldosteronism demographics in the Taiwan Primary Aldosteronism Investigation Group (TAIPAI Group). J Renin Angiotensin Aldosterone Syst. 2011;12(3):348-57. 
33. Wu VC, Kuo CC, Wang SM, Liu KL, Huang KH, Lin YH, Chu TS, Chang HW, Lin CY, Tsai CT, et al. Primary aldosteronism: changes in cystatin C-based kidney filtration, proteinuria, and renal duplex indices with treatment. J Hypertens. 2011;29(9):1778-86.

34. Wu VC, Yang SY, Lin JW, Cheng BW, Kuo CC, Tsai CT, Chu TS, Huang KH, Wang SM, Lin YH, et al. Kidney impairment in primary aldosteronism. Clin Chim Acta. 2011;412(15-16):1319-25.

35. Wu CH, Wu VC, Yang YW, Lin YH, Yang SY, Lin PC, Chang CC, Tsai YC, Wang SM, T group. Plasma aldosterone after seated saline infusion test outperforms captopril test at predicting clinical outcomes after adrenalectomy for primary aldosteronism. Am J Hypertens. 2019;32(11):1066-74.

36. Sedlazeck FJ, Rescheneder P, Smolka M, Fang H, Nattestad M, von Haeseler A, Schatz MC. Accurate detection of complex structural variations using single-molecule sequencing. Nat Methods. 2018;15(6):461-8.

37. Zhao M, Lee WP, Garrison EP, Marth GT. SSW library: an SIMD Smith-Waterman C/C++ library for use in genomic applications. PLOS ONE. 2013;8(12):e82138.

\section{Publisher's Note}

Springer Nature remains neutral with regard to jurisdictional claims in published maps and institutional affiliations.

- fast, convenient online submission

- thorough peer review by experienced researchers in your field

- rapid publication on acceptance

- support for research data, including large and complex data types

- gold Open Access which fosters wider collaboration and increased citations

- maximum visibility for your research: over $100 \mathrm{M}$ website views per year

At BMC, research is always in progress.

Learn more biomedcentral.com/submissions 